\title{
The Use of Contrast-Enhanced Transthoracic Echocardiography for Spiral Variant Hypertrophic Cardiomyopathy
}

\author{
Andrew Begg ${ }^{1}$, Garima Dahiya ${ }^{1}$, Andreas Kyvernitakis ${ }^{1}$, and Robert Biederman ${ }^{2}$ \\ ${ }^{1}$ Allegheny Health Network \\ ${ }^{2}$ Affiliation not available
}

July 2, 2020

\begin{abstract}
Hypertrophic cardiomyopathy is a common heritable cardiomyopathy with various clinical phenotypes. A rare spiral variant has been recently reported that has been associated with adverse outcomes and has traditionally been diagnosed using cardiac magnetic resonance. We report a case of the rare variant spiral hypertrophic cardiomyopathy where we used transthoracic echocardiography with an ultrasound enhancing agent to demonstrate the geometry of spiral hypertrophic cardiomyopathy and compared to simultaneous cardiac MRI images. The use of echocardiography with ultrasound enhancing agents may prove to be a valuable tool in identifying the geometry of hypertrophic cardiomyopathy variants in selected patients.
\end{abstract}

\section{Introduction}

Hypertrophic cardiomyopathy (HCM) is the most common heritable cardiomyopathy, characterized by left ventricular (LV) hypertrophy, in the absence of a secondary cause, with a typically asymmetric septal hypertrophy and mid-cavity obliteration phenotype $[1,2]$. Spiral HCM, a rare variant, with a counterclockwise rotation pattern of myocardial hypertrophy, typically mandates diagnosis by cardiac magnetic resonance (CMR) [3-7]. We report, to our knowledge, the first case of a spiral variant HCM visualized using routine transthoracic echocardiography (TTE). An ultrasound enhancing agent (UEA) allowed us to identify its rare geometric phenotype.

\section{Case presentation}

A 46 year old female with a reported history of HCM, but poor cardiology follow up, presented to the hospital with progressively worsening symptoms of congestive heart failure. Prior to presentation, she experienced flu-like symptoms for a week which were treated by her primary care physician with oral antibiotics and as needed albuterol inhaler. She reported an initial improvement of her symptoms, however, soon noted development of rapid-onset palpitations, presyncope, dyspnea on exertion, and lower extremity edema prior to presenting to the emergency department. On arrival she was hemodynamically stable but found to be in atrial fibrillation with rapid ventricular response. She was noted to have mild pulmonary edema, otherwise with a warm and well perfusing clinical profile, and oxygenating well on room air. She was admitted and started on rate control with a diltiazem infusion along with systemic anticoagulation and intravenous diuresis.

TTE obtained on hospitalization day 1 raised significant concerns for HCM given the findings of asymmetric basal septal hypertrophy with a maximum width of $2.2 \mathrm{~cm}$, hyperdynamic LV systolic function, and peak $\mathrm{LV}$ outflow tract (LVOT) gradient of $70 \mathrm{~mm} \mathrm{Hg}$ at rest. Systolic anterior motion of the anterior mitral valve leaflet and moderate mitral regurgitation were also noted.

Given the TTE findings, as well as episodes of non-sustained ventricular tachycardia on telemetry, a CMR was obtained on hospital day 3 to look for risk factors for sudden cardiac death (Figure 1). The latter revealed 
marked basal anterior LV hypertrophy with shifting geometry symmetrically towards the apex in a counterclockwise, spiral pattern, characteristic of spiral variant HCM (Figure 1). Late gadolinium enhancement (LGE) of greater than $20 \%$ of the myocardium was noted. Due to poorly tolerated atrial fibrillation, the patient was cardioverted back to normal sinus rhythm and was started on oral metoprolol to reduce inotropy and LVOT gradients. After successful cardioversion, a repeat TTE with the administration of (UEA) was performed on hospital day 6 to reassess the peak LVOT gradient and attempt to visualize the spiral variant HCM (Figure). The peak LVOT was noted to improve to $51 \mathrm{~mm} \mathrm{Hg}$ and the spiral HCM geometry was well visualized but only appreciated after administration of UEA to define the endomyocardial borders (Figure).

\section{Discussion}

HCM is a relatively common genetic cardiomyopathy that is believed to affect 1:500 people worldwide and an estimated 600,000 people in the United States [1]. HCM should be suspected in patients with maximal LV wall thickness of $>1.5 \mathrm{~cm}$ by echocardiography, in the absence of other compelling etiology [8]. The clinical presentation can vary from an incidental finding to clinically significant arrhythmias such as atrial fibrillation or sudden cardiac death, thereby making accurate diagnosis imperative [9].

Various HCM geometrical phenotypes have been reported, including more recently a rarely recognized spiralvariant, where there is segmental hypertrophy that spirals in a counter-clockwise pattern from the basal anterior septum towards the apex of the left ventricle $[4,10,11]$. Screening for HCM is traditionally accomplished with TTE with the addition of genetic testing for guidance regarding a definitive diagnosis as well as for screening recommendations for family members $[8,12]$. CMR is particularly helpful in the clarification of the diagnosis for patients with suboptimal echocardiographic windows, determining the HCM geometrical phenotype, and revealing the presence and mechanism of LVOT obstruction $[8,13]$ and risk for $\mathrm{SCD} / \mathrm{VT}$ as a function of percent scar $[7,8]$. It is clinically paramount to evaluate HCM patients for LVOT obstruction (defined as peak instantaneous gradient of [?] $30 \mathrm{~mm} \mathrm{Hg}$ ), as it is associated with poor prognosis and increased risk of sudden cardiac death [8].

While the benefits of CMR are clearly superior from a diagnostic and prognostic aspect in this population, TTE remains the preferred initial imaging modality for diagnosis of HCM [8]. The use of UEA in echocardiography is a technique that is used to enhance endocardial border delineation in studies with suboptimal left ventricular cavity visualization. This allows for improved sensitivities and specificities when comparing patients with poor echocardiographic windows to those with well visualized windows, and in rare occasions it may reveal previously occult yet ominous pathology [14-16]. Prior authors have suggested that echocardiography with 3D processing is also useful in visualizing the HCM geometric phenotype and pathophysiology of LVOT obstruction [17].

In our patient, we utilized a multi-modal approach, and although CMR provided a definitive diagnosis, the use of TTE with UEA allowed adequate visualization of the spiral HCM geometry which, to our knowledge we also represent as the first case diagnosed by TTE.

TTE findings of spiral variant HCM such as LVOT obstruction and systolic anterior motion of the mitral valve have been associated with worse clinical prognosis compared to non-spiral variants [18, 19]. Higher rates of non-sustained ventricular tachycardia and sudden cardiac death, as well as a higher incidence of heart failure and all-cause mortality have been reported with the spiral pattern of HCM [18].

In conclusion, the role of TTE has been to date restricted to screening patients for HCM with reliance on CMR for diagnosis and characterization of phenotype of HCM. In this case, we highlight that TTE with UEA may prove to be a valuable tool in demonstrating the geometry of the rare spiral variant HCM to the echocardiologist.

\section{References}

1. Geske JB, Ommen SR, Gersh BJ. Hypertrophic Cardiomyopathy: Clinical Update. JACC Heart Fail 2018;6(5):364-375. 
2. Klues HG, Schiffers A, Maron BJ. Phenotypic spectrum and patterns of left ventricular hypertrophy in hypertrophic cardiomyopathy: morphologic observations and significance as assessed by two-dimensional echocardiography in 600 patients. J Am Coll Cardiol 1995;26(7):1699-1708.

3. Amin N, Williams RB, Yarmozik JA, Biederman RW. Spiral hypertrophic cardiomyopathy as detected by cardiac magnetic resonance. Echocardiography 2014;31(3):E88-91.

4. Florian A, Masci PG, De Buck S et al. Geometric assessment of asymmetric septal hypertrophic cardiomyopathy by CMR. JACC Cardiovasc Imaging 2012;5(7):702-711.

5. Reichek N. Seeing spirals. JACC Cardiovasc Imaging 2012;5(7):712-714.

6. Higgins CB, Byrd BF, 3rd, Stark D et al. Magnetic resonance imaging in hypertrophic cardiomyopathy. Am J Cardiol 1985;55(9):1121-1126.

7. Moon JC, McKenna WJ, McCrohon JA, Elliott PM, Smith GC, Pennell DJ. Toward clinical risk assessment in hypertrophic cardiomyopathy with gadolinium cardiovascular magnetic resonance. J Am Coll Cardiol 2003;41(9):1561-1567.

8. Gersh BJ, Maron BJ, Bonow RO et al. 2011 ACCF/AHA guideline for the diagnosis and treatment of hypertrophic cardiomyopathy: executive summary: a report of the American College of Cardiology Foundation/American Heart Association Task Force on Practice Guidelines. J Am Coll Cardiol 2011;58(25):27032738 .

9. Nagueh SF, Bierig SM, Budoff MJ et al. American Society of Echocardiography clinical recommendations for multimodality cardiovascular imaging of patients with hypertrophic cardiomyopathy: Endorsed by the American Society of Nuclear Cardiology, Society for Cardiovascular Magnetic Resonance, and Society of Cardiovascular Computed Tomography. J Am Soc Echocardiogr 2011;24(5):473-498.

10. Reichek N, Gupta D. Hypertrophic cardiomyopathy: cardiac magnetic resonance imaging changes the paradigm. J Am Coll Cardiol 2008;52(7):567-568.

11. Masci PG, De Bondt J, Bogaert J. Helical form of hypertrophic cardiomyopathy: a new entity? Eur Heart J 2008;29(6):706.

12. Mandes L, Rosca M, Ciuperca D, Popescu BA. The role of echocardiography for diagnosis and prognostic stratification in hypertrophic cardiomyopathy. J Echocardiogr 2020.

13. Maron MS, Maron BJ, Harrigan C et al. Hypertrophic cardiomyopathy phenotype revisited after 50 years with cardiovascular magnetic resonance. J Am Coll Cardiol 2009;54(3):220-228.

14. Kurt M, Shaikh KA, Peterson L et al. Impact of contrast echocardiography on evaluation of ventricular function and clinical management in a large prospective cohort. J Am Coll Cardiol 2009;53(9):802-810.

15. Nanda NC, Kitzman DW, Dittrich HC, Hall G, Imagent Clinical Investigators G. Imagent improves endocardial border delineation, inter-reader agreement, and the accuracy of segmental wall motion assessment. Echocardiography 2003;20(2):151-161.

16. Siebelink HM, Scholte AJ, Van de Veire NR et al. Value of contrast echocardiography for left ventricular thrombus detection postinfarction and impact on antithrombotic therapy. Coron Artery Dis 2009;20(7):462466.

17. Erden M, van Velzen HG, Menting ME et al. Three-dimensional echocardiography for the assessment of left ventricular geometry and papillary muscle morphology in hypertrophic cardiomyopathy. J Ultrasound 2018;21(1):17-24.

18. Viliani D, Pozo E, Aguirre $\mathrm{N}$ et al. Helical distribution of hypertrophy in patients with hypertrophic cardiomyopathy: prevalence and clinical implications. Int J Cardiovasc Imaging 2017;33(11):1771-1780. 
19. Song Y, Yang DH, B OH et al. Geometric predictors of left ventricular outflow tract obstruction in patients with hypertrophic cardiomyopathy: a 3D computed tomography analysis. Eur Heart J Cardiovasc Imaging 2018;19(10):1149-1156.

Figure Legend: CMR (above) and TTE with UEA enhancement (below) short axis images of the base, mid, and apical left ventricle. Arrows are pointing towards the typical basal anterior wall to apical counterclockwise rotational hypertrophy pattern seen in patients with spiral HCM.
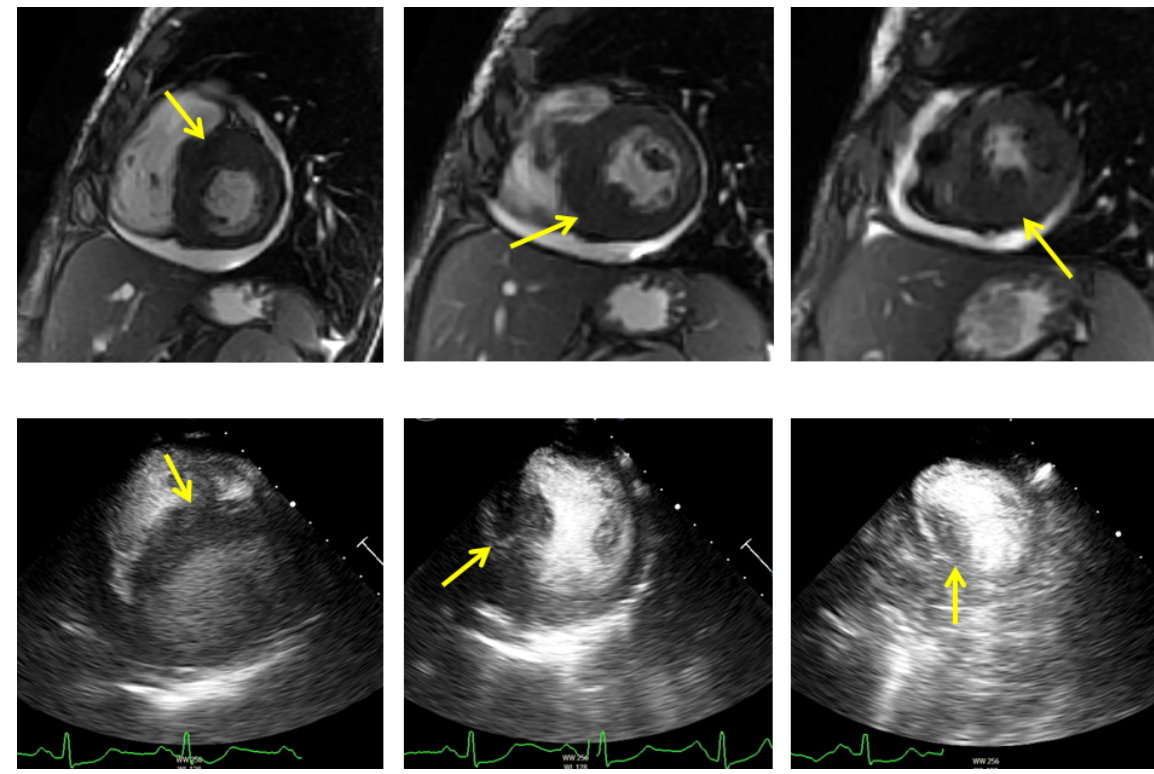